\title{
Voice Morphology, Case, and Argument Structure in Malagasy
}

\author{
Matt Pearson \\ Reed College
}

\section{Introduction}

The canonical Malagasy clause is divided into two constituents, the predicate phrase (PredP), and a definite noun phrase here called the trigger. This is schematized in (1) (cf. Keenan 1995 for evidence for this bipartite clause structure).

$$
\text { [PredP V ...] [Trigger DP ] }
$$

When the predicate phrase is headed by a verb, that verb is marked for voice to indicate the grammatical function of the trigger. Examples are given in (2) (here and throughout, the trigger is underlined; the morphological breakdown of each verb is given in brackets, with the voice morphemes boldfaced). (2a) illustrates the actor-trigger (AT) form, used when the external argument-here, the agent-is the trigger of the clause. The theme-trigger (TT) form in (2b) is used when an internal argument-here, the patient-functions as the trigger. Finally, the circumstantial-trigger (CT) form in (2c) is used when the trigger is an oblique element-here, the instrument with which the event is carried out. In each case, the trigger follows the predicate phrase, occurring at the right periphery of the sentence. Within the predicate phrase, the order of elements is VSOX: non-trigger external arguments appear right-adjacent to the verb, preceding internal arguments, which precede obliques.

(2) a. Mamono [ $\mathbf{m}-a N$-vono ] akoho amin'ny antsy ny.....mpamboly. AT.kill m-Pfx-kill chicken with-Det knife Det farmer 'The farmer kills chickens with the knife'

b. Vonoin' [ vono-in ] ny mpamboly amin'ny antsy ny ....akoho TT.kill kill-in Det farmer with-Det knife Det chicken 'The farmer kills the chickens with the knife'

c. Amonoan' [ $a N$-vono-an ] ny mpamboly akoho ny.....antsy CT.kill Pfx-kill-an Det farmer chicken Det knife 'The farmer kills chickens with the knife'

In previous work (Pearson, to appear), I used evidence from binding, extraction, and other domains to argue that the trigger is not the subject of the sentence, as usually assumed, but is instead base-generated in the specifier of an A'-projection, TopicP, and linked to a null operator in the specifier of a lower projection, $W h P$, as shown in (3) (abstracting away from surface word order; on the rightperipheral position of the trigger, see Pearson (2001)).

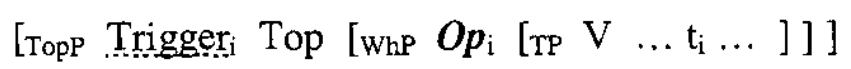

The analysis in (3) suggests a novel approach to the voice alternations in (2): Generally, voice morphology is taken to encode active/passive-like alternations in 
the mapping of thematic roles to the subject (nominative Case-checking) position. However, if the trigger is not the subject of the clause but a topic linked to an A'chain, it is possible that voice morphology instead indicates the position from which A'-extraction takes place, with the AT form in (2a) marking subject extraction, the TT form in (2b) marking object extraction, and the CT form in (2c) marking oblique extraction.

Under this approach, voice in Malagasy is less like voice in English and more like wh-agreement, of the sort which Chung (1998) documents for Chamorro. In A'-extraction contexts in Chamorro, regular subject agreement of the sort shown in (4) is replaced by special morphology indicating whether the extracted element is a subject, object, or oblique, as in (5). In Pearson (to appear) I suggested that Malagasy voice marking is a 'generalized' version of this type of marking: While in Chamorro wh-agreement is confined to questions, relative clauses, and the like, in Malagasy it appears in all clause types due to a requirement that the specifier of WhP be filled in every clause.

(4) Ha-fa'gasi si Juan i kareta

3s-wash Det Juan Det car

'Juan washed the car'

(5) a. Hayi f<um $>$ a'gasi i kareta?

who um-wash Det car

'Who washed the car?'

b. Hafa $\mathrm{f}<\mathrm{in}>\mathrm{a}$ 'gasése-nña si Henry pära hagu? (obj. extraction) what in-wash.Prog-3s.Lnk Det Henry for you

'What is Henry washing for you?'

c. Hafa pära fa'gase-mmu ni kareta?

what Fut $\boldsymbol{\varnothing}$-wash-2s Obl car

(subj. extraction)

'What are you going to wash the car with?'

In this paper I focus on the voice affixes themselves and propose an account of their distribution. Specifically, I argue that they are realizations of light verbs and Case-checking heads, which combine with the root through head-to-head movement. The distribution of the affixes is determined by the positions from which, and through which, the null operator in (3) moves on its way to the specifier of WhP. For example, the actor-topic prefix $m$ - is treated as a nominative Casechecking head, which gets spelled out just in case the operator raises through its specifier. (My analysis is thus in the spirit of Guilfoyle, Hung, \& Travis (1992), who also associate voice morphemes with Case licensing.)

\section{The Morphology of voice}

Before proceeding with this analysis, I offer a quick overview of Malagasy voice morphology. Verbs in Malagasy are formed from roots, some of which function independently in the language, either as nouns or as stative predicates. To form a verb stem, the root combines with one of a small set of verbal prefixes (glossed 
'Pfx' in the examples), of which the two most common are $a N-$ and $i$-. Some examples of roots and the verb stems formed from them are given in (6).

$\begin{array}{llll}\text { Root } & & \text { VERB STEM } & \\ \text { asa } & \text { 'work, task' } & i \text {-asa } & \text { 'work' } \\ \text { fefy } & \text { 'enclosure' } & i \text {-fefy } & \text { 'be fenced' } \\ \text { vavaka } & \text { 'prayer' } & i \text {-vavaka } & \text { 'pray' } \\ \text { feno } & \text { 'full' } & a N \text {-feno [ameno] } & \text { 'fill' } \\ \text { soratra } & \text { 'writing' } & a N \text {-soratra [anoratra] } & \text { 'write' } \\ \text { tapaka } & \text { 'broken' } & a N \text {-tapaka [anapaka] } & \text { 'break' }\end{array}$

Although the choice between $a N$ - and $i$ - is lexically determined, it does correlate to some degree with transitivity: $a N$ - stems are almost always transitive, while $i$ stems tend to be intransitive. As illustrated in (7), there are a large number of roots which can take either prefix, where $a N$ - forms a transitive stem and $i$ - forms its intransitive counterpart:

$$
\begin{array}{llll}
i \text {-haja } & \text { 'be respected' } & a N \text {-haja [anaja] } & \text { 'respect (tr.)' } \\
i \text {-sasa } & \text { 'wash oneself' } & a N \text {-sasa [anasa] } & \text { 'wash (tr.)' } \\
i \text {-voha } & \text { 'be open' } & a N \text {-voha [amoha] } & \text { 'open (tr.)' }
\end{array}
$$

Additional affixes are attached to roots and stems to mark voice. There are five morphologically distinct voice forms in Malagasy, listed in the table below an illustrated using $a N$-tafi 'wrap, dress' and aN-velar 'unroll, spread out' (two of a handful of stems which can occur in all five forms). The actor-trigger (AT) voice is formed by prefixing $m$ - to the stem; while the circumstantial-trigger (CT) voice is formed with the suffix -an. The other three voices are usually grouped together as the theme-topic (TT) voices. These are formed by adding the suffix -an or -in,

\begin{tabular}{|c|c|c|c|c|c|}
\hline VOICE & \multicolumn{3}{|c|}{ TEMPLATE } & \multicolumn{2}{|l|}{ EXAMPLES } \\
\hline$\overline{\mathrm{AT}}$ & $\mathrm{m}-\quad P f x$ & ROOT & & $\begin{array}{l}\text { m- } a N \text {-tafi } \\
\text { m- } a N \text {-velar }\end{array}$ & $\begin{array}{l}>\text { manafy } \\
>\text { mamelatra }\end{array}$ \\
\hline $\mathrm{CT}$ & $P f x$. & ROOT & -an & $\begin{array}{l}a N \text {-tafi-an } \\
a N \text {-velar-an }\end{array}$ & $\begin{array}{l}>\text { anafiana } \\
>\text { amelarana }\end{array}$ \\
\hline \multirow[t]{3}{*}{$\mathrm{TT}$} & & ROOT & -an & $\begin{array}{l}\text { tafi-an } \\
\text { velar-an }\end{array}$ & $\begin{array}{l}>\text { tafiana } \\
>\text { velarana }\end{array}$ \\
\hline & & ROOT & -in & $\begin{array}{l}\text { tafi-in } \\
\text { velar-in }\end{array}$ & $\begin{array}{l}>\text { tafina } \\
>\text { velarina }\end{array}$ \\
\hline & a- & ROOT & & $\begin{array}{l}\text { a-tafi } \\
\text { a-velar }\end{array}$ & $\begin{array}{l}>\text { atafy } \\
>\text { avelatra }\end{array}$ \\
\hline
\end{tabular}
or the prefix $a$-. Notice that the verbal prefix is absent in the TT forms, a fact to which I return in the next section. ${ }^{1}$

Examples of these five forms are given in (8)-(10). The AT form is used when the trigger is the subject of the clause--that is, the sole 'core' argument of an intran- 
sitive verb (8a), or the external argument of a transitive verb (8b). The TT voices are used when the trigger is the direct or indirect object of a transitive verb (9) (see sections 4 and 5 for more on the distribution of these affixes). ${ }^{2}(10)$ illustrates the CT form, which is used when the trigger bears some oblique role such as instrument, location, or benefactee.

(8) a. Mipetraka [ $\mathbf{m}-i$-petrak ] any Antsirabe ny vehivavy

AT.live $\mathbf{m}$ - $P f x$-live there Antsirabe Det woman

'The woman lives in Antsirabe'

b. Manoratra [ $\mathbf{m}-a N$-sorat ] taratasy ny .....mpianatra

AT.write $\mathbf{m}$-Pfx-write letter Det student

'The student is writing a letter'

(9) a. Tapahin' [ tapak-in ] ny lehilahy ny...... vahitadgdy.

TT.cut cut-in Det men Det vine.rope

'The men cut the vine rope'

b. Soratan' [ sorat-an] ny mpianatra ny taratastasy

TT.write write-an Det student Det letter

'The student is writing the letter'

c. Atao [a-taov ] ny.... fiomanana rehetra

TT.make a-make Det preparation all

'All the preparations are being made'

(10) a. Amonoan' [ $a N$-vono-an ] ny mpamboly akoho ny antsy

CT.kill Pfx-kill-an Det farmer chicken Det knife

'The farmer kills chickens with the knife'

b. Itoeran' [ $i$-toer-an ] ny ankizy io t...trano io

CT.live Pfx-live-an Det children this house this

'The children live in that house'

c. Amonoan' [ $a N$-vono-an] ny mpamboly akoho ny..... vahiny.

CT.kill Pfx-kill-an Det farmer chicken Det guest

'The farmer is killing chickens for the guestss'

I now consider each of the morphemes in the above table in turn. I start with the verbal prefixes, and then turn to the AT prefix $m$ - and the TT suffix -in. Next I consider the TT prefix $a$-. Finally, I discuss $-a n$, found in the remaining TT form, and in the CT form.

\section{The verbal prefixes}

As shown in the table above, the verbal prefixes $a N$ - and $i$-appear in AT clauses, where the subject is extracted, and in CT clauses, where an oblique element is extracted. However, these prefixes are absent in TT clauses, where an object is extracted. What's the reason for this? Recall that $a N$ - and $i$-form verb stems from roots, many of which can occur independently: For instance, (12) shows that $a \mathrm{~N}$ may attach to a one-place stative root to form a two-place verb, adding an agent argument in the process. 
(11) a. Feno ny tavoahangngy

full Det bottle

'The bottle is full'

b. Mameno [ $\mathbf{m}-a N$-feno] ny tavoahangy ny vehivavy.

AT.fill m-Pfx-full Det bottle Det woman

'The woman is filling the bottle'

Thinking in terms of a Hale \& Keyser approach to argument structure, this suggests that the verbal prefixes are light verbs, which take a root or one-place predicate as their complement, and project an external argument in their specifier. This is schematized in (12) (here and below, I abstract away from head-to-head movement; I assume that verb adjoins successively to each head in its extended projection, raising as high as $\mathrm{T}$ before Spell Out). Assuming that the prefixes are light verbs (and assuming furthermore that obliques are merged outside of $\nu \mathrm{P}$; cf. section 6), the proper generalization is as follows: The light verb $v$ is suppressed (rendered covert) when an operator undergoes A'-extraction from its complement.

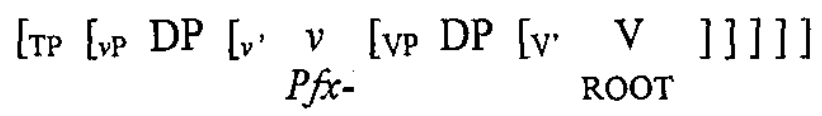

The connection between extraction and the spell-out of the light verb receives a straightforward explanation in terms of phases. In his account of successive cyclic movement, Chomsky $(1999,2000)$ divides the clause into two domains, a lower phase, identified with $v \mathrm{P}$, and an upper phase, identified with CP. In accordance with the Phase Impenetrability Condition (13) (cf. Chomsky 2000, p. 108), a constituent can move out of a phase only by first moving to its left edge, merging as a specifier of the highest head in the phase. If this is correct, then in order for an operator to extract from the complement of the light verb in Malagasy, it must first raise to become a specifier of $\nu \mathrm{P}$, as shown in (14). In other words, Spec $\nu \mathrm{P}$ is an escape hatch for $\mathrm{A}^{\prime}$-movement.to SpecWhP in TT clauses:

(13) In phase $\alpha$ with head $\mathrm{H}$, the domain of $\mathrm{H}$ is not accessible to operations outside $\alpha$; only $\mathrm{H}$ and its edge (i.e., the specifier(s) of HP) are accessible to such operations.

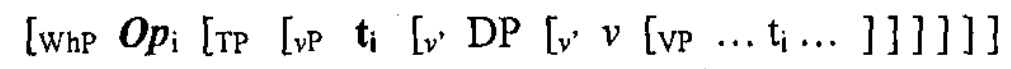

I propose that the prefixes $a N$ - and $i$ - alternate with a $\varnothing$ realization of the light verb. The empty light verb has a feature which attracts an operator into its specifier. By contrast, the overt light verbs $a N$ - and $i$ - lack this feature, and so may not host an operator. In other words, extraction from the lower phase is possible only when the head of the lower phase $v$ is spelled out as the $\emptyset$ allomorph; when $v$ is an overt light verb, this renders it impenetrable for A'-extraction.

Alternations of this sort are well known in the head of the upper phasethat is, the complementizer head. In languages such as Irish, for example, the form of the complementizer depends in part on whether there is an $\mathrm{A}^{\prime}$-operator in the specifier of CP. The usual finite complementizer is go, as shown in (15); how- 
ever, when an operator raises to (or through) SpecCP, the complementizer $a$ is used instead, as shown in (16). I suggest that the alternation in Malagasy between the verbal prefixes $a N$ - or $i$ - and $\varnothing$ is a lower phase analogue of this complementizer alternation: In each case, the availability of a landing site or escape hatch for A-bar movement correlates with how the head of the phase is realized. (Examples taken from Carnie, Harley, \& Pyatt 2000, Guilfoyle 2000, and Chung \& McCloskey 1987 , respectively.)

$$
\begin{aligned}
& \text { Ceapaim [ go bhfaca sé an madra ] } \\
& \text { think.1s Comp saw he the dog } \\
& \text { 'I think that he saw the dog' }
\end{aligned}
$$

(16) a. Caidé a chuir sin i do cheann? what Comp put that in your head 'What put that in your head?'

b. an bhean [OP a chuir t-isteach air ] the woman Comp put in on.it 'the woman who applied for it'

\section{Intransitive and monotransitive verbs}

Next I turn to the AT prefix $m$ - and the TT suffix -in. Recall that $m$ - appears on the verb when the transitive or intransitive subject is the trigger, as in (17) and (18a), while -in appears when the transitive object is the trigger, as in (18b):

$$
\begin{aligned}
& \text { Mitomany [ m-i-tomani ] ny.... zazavayy } \\
& \text { AT.cry m-Pfx-cry Det girl } \\
& \text { 'The girl is crying' }
\end{aligned}
$$

(18) a. Mamaky [ $\mathbf{m}-\mathrm{aN}$-vaki ] boky ny ....zazavavy.

AT.read m-Pfx-read book Det girl

'The girl is reading a book'

b. Vakin' [ vaki-in ] ny zazavavy ny .....oky.

TT.read read-in Det girl Det book

'The girl is reading the book'

Assuming that Malagasy is a nominative-accusative language, it seems that AT marking correlates with nominative Case, while TT marking correlates with accusative Case. I propose that $m$ - and $-i n$ are realizations of the functional heads which license nominative and accusative Case, respectively: When the operator which raises to SpecWhP has a nominative Case feature to check, the head which checks it is spelled out on the verb as $m$-, and when the operator has an accusative Case feature, the head which checks that feature is spelled out as $-i n$.

First of all, consider the sentences in (19) and (20), featuring a TT verb prefixed with $a$-. As these examples show, the predicate-internal subject may be overt, as in the (b) sentences; or it may be covert, as in the (a) sentences, in which case the agent is construed as unknown or arbitrary. Notice that overt subjects im- 
mediately follow the verb, which is suffixed with the linking morpheme, glossed 'Lnk' in the examples (this linking morpheme is realized as $-y$ between two noncontinuant consonants, otherwise as $-n$ '; I assume that the underlying form of this morpheme is $-n y)$.

(19) a. Atosika [a-tosek ] ny .....vato

TT.push a-push Det stone

'Someone is pushing the stone' or 'The stone is being pushed'

b. Atosiky [a-tosek-ny ] ny vehivavy ny..... vato

TT.push a-push-Lnk Det woman Det meal

'The woman is pushing the stone'

(20) a. Aroso [ a-roso ] ny vahiny ny s...sakafọo

TT.serve a-serve Det guest Det meal

'Someone serves the guests the meal' or 'The meal is served to the guests'

b. Aroson' [ a-roso-ny ] ny vehivavy ny vahiny ny ....sakafọ

TT.serve a-serve- $\boldsymbol{L} \boldsymbol{n} \boldsymbol{k}$ Det woman Det guest Det meal

'The woman is serving the guests the meal',

Although the linking morpheme surfaces only on stems taking the $a$ - prefix, I will assume that it is also present on stems ending in -in and -an when they have overt subjects, but fails to surface because of fusion with the $n$ of the voice suffix:

(21) a. Vakina [ vaki-in ] no...... boky.

TT.read read-in Det book

'The book is being read'

b. Vakin' [ vaki-in-ny ] ny zazavavy ny.... boky.

TT.read read-in-Lnk Det girl Det book

'The girl is reading the book'

Since the linking morpheme is required to license an overt subject in the predicate phrase, I will assume that it is located in the functional head which checks nominative Case. Adapting an analysis due to Travis (1994), I identify this as the event head $\mathrm{E}$, which selects $v \mathrm{P}$ as its complement, as shown in (22). In addition to checking nominative Case, the event head introduces (or binds) the event argument of the clause, and is in turn selected by the tense head. As an examination of the forms above shows, the linking morpheme is in complementary distribution with the AT prefix $m-:$ I will therefore assume (following Travis (1994)) that the $m$ - prefix is also in E. So $m$ - and $-n y$ are alternate realizations of the nominative Case-checking head.

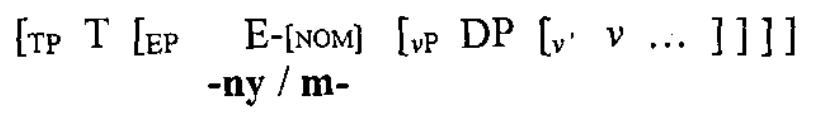

What determines how $\mathrm{E}$ will be realized? Given my theory of triggers, this correlates with whether the subject (i.e., the nominative Case-marked argument) undergoes A'-movement or not: When the subject is an operator which raises to 
SpecWhP, the verb carries the prefix $m$-, and when the subject remains inside TP, the verb carries the suffix $-n y$. To capture this alternation, I propose that how the $E$ head is realized depends on whether or not it contains an EPP feature in addition to its Case feature-that is, whether or not it projects a specifier, triggering raising of the nominative DP (cf. Chomsky 1999, 2000): When E carries an EPP feature, it is spelled out as $m$-, and when it lacks an EPP feature, it is spelled out as $-n y$. I will refer to a head containing an EPP feature as strong, and a head which lacks an EPP feature as weak.

When the subject is an operator which raises to SpecWhP to check a feature of Wh, it must pass through the specifier of EP in order to check its Case feature. Consequently, E will have to be strong, and so it will be spelled out as $m$-. The structure is shown in (23) (abstracting away from head-to-head movement). However, when the subject does not raise to SpecWhP, it does not need to pass through SpecEP in the overt syntax. In principle E could strong or weak in such cases. Suppose that economy dictates in favor of the weak form, and so the verb carries the suffix -ny. In this case, the subject is spelled out in its base position, Spec $v \mathrm{P}$, as in (22), and checks its Case via an Agree relation, without movement.

$$
\begin{aligned}
& {\left[\mathrm { WhP } O \boldsymbol { p } _ { \mathrm { i } } \left[\mathrm { TP } \left[\mathrm { EP } \mathrm { t } _ { \mathrm { i } } \left[\mathrm{E}, \mathrm{E}\left[\begin{array}{llllll}
v \mathrm{P} & \left.\left.\mathrm{t}_{\mathrm{i}}\left[\begin{array}{lllll}
v & v & \ldots & \mathrm{V} & ]
\end{array}\right]\right]\right]
\end{array}\right]\right.\right.\right.\right.} \\
& \text { m- } \quad P f x-\quad \text { ROOT }
\end{aligned}
$$

My analysis of the TT suffix -in takes essentially the same form. Alongside the nominative Case-checking head E, I assume there is a second functional head responsible for checking accusative Case, located between the light verb and the root. Following Travis (1991), I identify this as the aspect head, which projects an AspP, selected as the complement of the light verb, as in (24).

$$
\left[v \mathrm{P}(\mathrm{DP})\left[v^{,} \underset{(P f x-)}{v} \underset{\varnothing}{[\mathrm{AspP}} \operatorname{Asp-[\mathrm {AcC}]}\left[\mathrm{vP} \text { DP }\left[\begin{array}{lll}
\mathrm{v}^{\prime} & \mathrm{V}
\end{array}\right]\right]\right]\right]
$$

Like the event head, the aspect head is spelled out differently depending on whether it is strong or weak. When it is strong, and triggers raising of the object for accusative Case checking, it is spelled out as -in; otherwise it surfaces as $\varnothing$. When the verb is intransitive, and Asp lacks an accusative Case feature altogether, the $\varnothing$ variant will be selected. When the verb is transitive, either variant may be selected. Normally the $\varnothing$ variant will be selected, and the direct object will remain in situ, checking its Case feature without movement, as in (24). However, when the direct object is an operator, its Case is checked via overt movement to SpecAspP. In such cases the aspect head must be strong, and so the suffix -in will be inserted. From SpecAspP the operator raises to the edge of the lower phase, attracted by the phonetically empty light verb, before raising out of the lower phase to SpecWhP. This is summarized in (25):

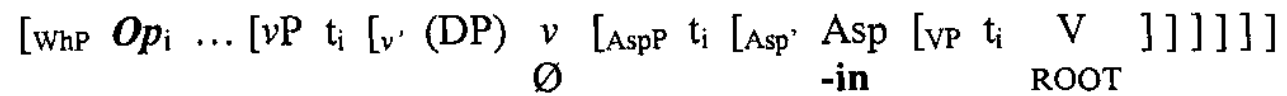




\section{Ditransitive constructions}

Although the majority of monotransitive verbs mark the TT voice using the suffix $-i n$, there are a number of verbs which take the suffix - an instead, or the prefix $a$-, as illustrated in (9) above. More often, however, $-a$ and $a$ - are used with ditransitive verbs. As Paul (1999) discusses, ditransitives in Malagasy fall into various semantic classes: One class, illustrated by roso 'serve' in (26), includes verbs of transmission, selecting a goal or recipient and a locatum: Here $-a n$ is used when the goal is the trigger (26b), while $a$ - is used when the locatum is the trigger (26c). A second class of ditransitives is illustrated by didi 'cut' in (27): This class includes verbs which select a patient, and an additional argument denoting an instrument acted upon by the agent to bring about a change in the patient. With verbs of this type, -an is used when the patient is the trigger (27b), while $a$ - is used when the instrument is the trigger (27c):

(26) a. Mandroso [ $\mathbf{m}$-aN-roso ] sakafo ny vahiny ny....mpamboly AT.serve m-Pfx-serve meal Det guest Det farmer 'The farmer serves the guests a meal'

b. Rosoana [ roso-an ] sakafo ny.....yahiny. TT.serve serve-an meal Det guest 'The guests are served a meal'

c. Aroso [ a-roso ] ny vahiny ny sakafono TT.serve a-serve Det guest Det meal 'The meal is served to the guests'

(27) a. Mandidy [ $\mathbf{m}$-aN-didi ] antsy ny hena ny mpamboly AT.cut m-Pfx-cut knife Det meat Det farmer 'The farmer cuts the meat with a knife'

b. Didiana [ didi-an ] antsy ny hena TT.cut cut-an knife Det meat 'The meat is cut with the knife'

c. Adidy [ a-didi ] ny hena ny antsy. TT.cut a-cut Det meat Det knife 'The knife is used to cut the meat'

The verbs which take both -an and $a$ - TT forms constitute the double object verbs of Malagasy - that is, those verbs which allow two internal DP arguments. Comparing the sentences in (26) above, we see that the trigger of the $a$-clause in (c) corresponds to the leftmost object in the actor-topic sentence in (a) (usually indefinite and adjacent to the verb), while the trigger of the -an clause in (b) corresponds to the rightmost object in (a). This pattern holds for (27). Following Dryer (1986), I will refer to the object farther from the verb as the primary object (PO), and the object closer to the verb as the secondary object (SO), as in (28). Hence the proper generalization is that the -an form indicates that the PO of a double object construction has undergone raising to SpecWhP, while the $a$ - form indicates that the $\mathrm{SO}$ has undergone raising. 
(28)

$\begin{array}{llll} & \text { SO } & \text { PO } & \\ \text { Mandroso } & \frac{\text { sakafo }}{\text { meal }} & \frac{\text { ny vahiny }}{\text { Det guest }} & \text { ny mpamboly } \\ \text { AT.serve farmer } \\ \text { 'The farmer serves the guests a meal' } & \\ \text { Mandidy } \frac{\text { antsy }}{\text { knife }} & \frac{\text { ny hena }}{\text { Det meat }} & \text { ny mpamboly } \\ \text { AT.cut } & \text { Det farmer } \\ \text { 'The farmer cuts the meat with a knife' } & \end{array}$

Following Marantz (1993), I assume that double object constructions are really applicative constructions in which the applicative morpheme is null. As schematized in (29) below, Marantz treats the applicative morpheme as a kind of light verb, which selects a phrase containing the lexical root as its complement (cf. Larson 1988). The PO merges as the specifier of the applicative head, while the SO merges within its complement. Based on data from Bantu applicatives, Marantz argues that the structure in (29) compositionally represents the complex event denoted by an applicative construction, such that the PO is interpreted as affected by the sub-event denoted by the complement of the applicative head. Hence, for verbs taking a goal and a locatum, it is the goal which will be mapped to the PO function, while verbs taking a patient and an instrument will map the patient to the PO function.

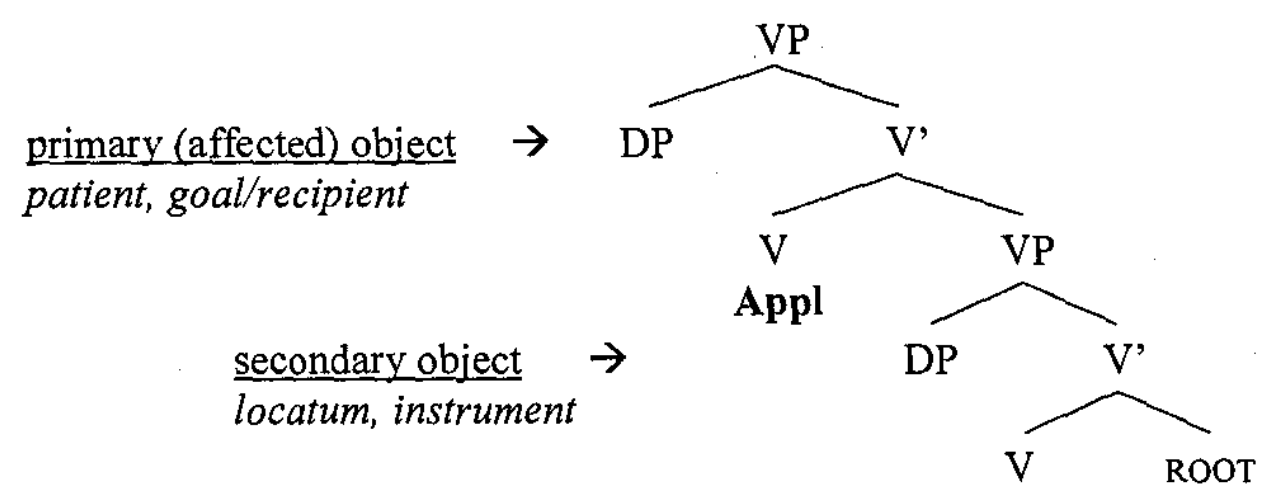

Under this analysis, the $a$ - prefix receives a similar treatment to $m$ - and -in, discussed earlier. Suppose that in double object constructions, the Case feature of the PO is checked by the Asp head, which selects the structure in (29); while the Case feature of the SO (partitive?) is checked by a lower functional head F, which takes the VP containing the root as its complement and projects an FP, selected as the complement of the applicative head. As with $\mathrm{E}$ and Asp, assume that the $\mathrm{F}$ head may be strong or weak, where the prefix $a$-spells out a strong $\mathrm{F}$ head. When the SO is an operator attracted to SpecWhP, its Case is checked in Spec of FP by a strong $\mathrm{F}$, and the prefix $a$ - appears on the verb; otherwise the SO remains in situ, and the F head is null. The structure is given in (30): The SO raises first to SpecFP to check Case. It then raises further to the edge of the lower phase (forcing $v$ to be null) before raising on to SpecWhP.

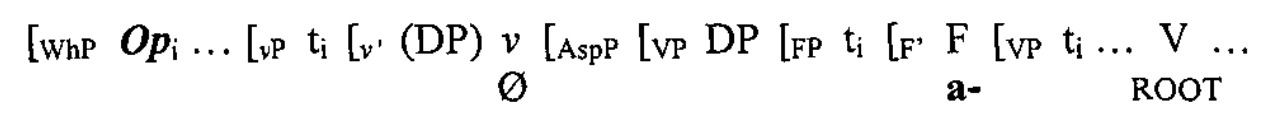


As for -an, I treat this suffix as the applicative morpheme in (29). When the operator in SpecWhP raises from the PO position, the applicative head which takes the PO as its specifier is spelled out as -an, as schematized in (31). When the PO remains in situ, the applicative head is null. ${ }^{3}$

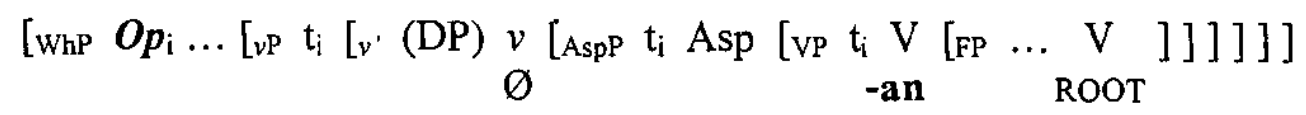

In support of treating -an as a spell-out of the applicative morpheme, observe the following: First, it seems that a ditransitive verb in Malagasy accepts the -an TT form if and only if it can occur in a double object construction. Consider the verb stem $a N$-petrak 'put' in (32), which arguably selects two arguments, a locatum and a goal. As shown in (32b), 'put' does not license a double object construction; rather, the goal must take the form of a PP, as in (32a). (32c) shows that, as expected, the -an TT form is also disallowed. This is presumably because the goal of a putting event is not normally understood to be affected by the action.

(32) a. Mametraka [ m-aN-petrak ] boky eo ambonin'ny seza ny...zaza
AT.put mair Det child
'The child is putting books on the chair'
b. *Mametraka [ m-aN-petrak ] boky ny seza ny...zaza
AT.put book Det chair Det child
'The child is putting books on the chair'
c. *Petrahana [ petrak-an] boky ny....seza
TT.put put-an book Det chair
'That chair is having books put on it'

On the other hand, the -an TT form can sometimes be used to convert an intransitive stem into a transitive stem by 'promoting' a locative adjunct to the role of affected object. Consider the stem i-petrak 'sit', the intransitive counterpart of aN-petrak: Being intransitive, this stem would not be expected to take TT morphology. However, my consultant allows the -an TT form when the location ny seza 'the chair' is the trigger, as in (33b)-but crucially only if the chair is understood as affected by the act of sitting on it; otherwise the CT form would be used. I take the patterns in (32) and (33) as evidence that the TT form with -an marks A'-extraction of an affected object in an applicative construction.
(33) a. Mipetraka [ $m$-i-petrak ] amin'ilay seza ny AT.sit m-Pfx-sit on-that chair Det child
'The child is sitting on that chair'
b. Petrahana [ petrak-an ] ilay ...seza
TT.sit sit-an that chair
'That chair is being sat in'

One question remains, namely: Why is -an is spelled out only if its specifier raises out? Here I follow Sportiche (1992) in adopting a generalized version of the 
'doubly-filled COMP' filter (34). In accordance with this filter, -an is spelled out when its specifier contains a trace; otherwise the applicative head is null. Note that (34) is meant to hold generally: As an examination of the structures in this paper will show, each of the projections I posit (with one exception, which I return to below) has an empty head, an empty specifier, or both, at Spell Out.

$$
\text { If } \mathrm{H} \text { is a head containing some feature } \mathrm{F},{ }^{*}\left[\mathrm{HP} X \mathrm{XP}\left[\mathrm{H}, \mathrm{H}^{0} \ldots\right]\right] \text { when } \mathrm{XP}
$$
and $\mathrm{H}^{0}$ both overtly encode $\mathrm{F}$.

\section{The circumstantial voice}

I have now provided analyses of the AT form and the three TT forms in the table in section 2. This leaves the CT voice, used when the trigger is an oblique-that is, an element which requires a preposition in clauses where it does not function as the trigger. Examples are given in (35b) and (36b), showing CT clauses with locative and instrumental triggers, respectively.

$$
\begin{aligned}
& \text { (35) a. Mitoetra [ } \mathbf{m}-i \text {-toer ] ao amin'io trano io ny...ankizy. } \\
& \text { AT.live m- mfx-live here in-this house this Det children } \\
& \text { 'The children live in that house' } \\
& \text { b. Itoeran' [ } i \text {-toer-an-ny ] ny ankizy io trano io } \\
& \text { CT.live Pfx-live-an-Lnk Det children this house this } \\
& \text { 'The children live in that house' }
\end{aligned}
$$

(36) a. Mamono [ $\mathbf{m}-a N$-vono ] akoho amin'ny antsy ny mpamboly. AT.kill m-Pfx-kill chicken with-Det knife Det farmer 'The farmer kills chickens with the knife'
b. Amonoan' [ $a N$-vono-an-ny ] ny mpamboly akoho ny.....antsy.
CT.kill 'The farmer kills chickens with the knife'

Notice that the CT form does not include any new morphemes, but rather a combination of morphemes found in other voice forms. Like the TT forms, the CT takes the linking morpheme -ny, indicating that the $\mathrm{E}$ head is weak and the subject is Case-licensed in SpecvP. The CT also includes the suffix -an, which means that the operator raises from the specifier of an applicative head. But unlike the TT form with -an, the root carries a verbal prefix. Since by assumption the lower phase is opaque to A'-extraction when a verbal prefix is present, it follows that the operator in SpecWhP in a CT clause has raised from somewhere outside of $v \mathrm{P}$.

Putting these pieces together, I conclude that the applicative head in Malagasy may be introduced in either the lower phase or the higher phase. In 'low' applicatives, the applicative head merges with the VP containing the root. Its specifier is a DP which checks accusative Case under agreement with the Asp head, and is interpreted as an 'affected object'. In 'high' applicatives, by contrast, the applicative head merges with some larger projection, possibly $v \mathrm{P}$. Its specifier does not contain a DP bearing accusative Case and interpreted as affected, but rather a PP. A possible structure is given in (37). ${ }^{4}$ 
Extraction of an operator from this higher applicative position yields the combination of morphemes associated with the CT voice, as shown in (38): The subject and object remain in situ, their Case-licensing heads spelled out as -ny and $\varnothing$, respectively. The operator raises from the specifier of the higher applicative head, causing its head to be spelled out as -an, in accordance with the 'doubly-filled COMP' filter. No extraction takes place from $v \mathrm{P}$, and so the light verb surfaces as a prefix on the verb stem. To explain the absence of a preposition in CT clauses, we may assume (a) that the operator is of category PP, and/or (b) that the oblique operator in SpecWhP (comparable to dont in French) does not need Case.

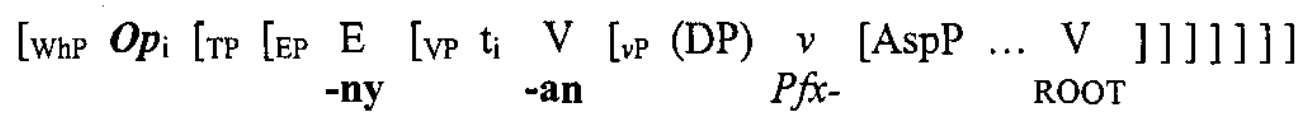

One apparent problem with (38) is that the $v \mathrm{P}$ projection violates the 'doubly-filled comP' filter discussed above: The head of $v \mathrm{P}$ is spelled out as a verbal prefix, while the specifier of $v \mathrm{P}$ contains the in situ subject. There are various possible solutions to this problem. For example, we could simply expand the tree so that the subject and the verbal prefix end up in different projections. A second, perhaps more interesting possibility is that $v \mathrm{P}$, by virtue of being a phase, is somehow exempt from the 'doubly-filled CoMP' filter. Adopting Chomsky's (1999) idea that Spell Out operates cyclically, we might speculate that the 'doubly-filled COMP' filter (plausibly a condition on Spell Out) applies only within a given cycle. Suppose that the head of a phase and its specifier are spelled out in different cycles, then it follows that $\nu \mathrm{P}$ would be able to have an overt head cooccurring with an overt specifier.

\section{Conclusion}

In this paper I have argued that the morphemes which combine to form the different voices in Malagasy are realizations of heads which attach to the root via headto-head adjunction in the syntax. The verb stem-forming prefixes are light verbs, which alternate with a null variant when $A^{\prime}$-extraction from the lower phase takes place. The AT prefix $m$-, the TT prefix $a$-, and the TT suffix -in are strong Caselicensing heads which surface just in case an operator raises through their specifiers. Finally, the suffix -an, found on the CT and on one of the TT forms, is an applicative head, which surfaces when the applicative argument raises out of its specifier, in accordance with a generalized 'doubly-filled COMP' filter.

\section{Endnotes}

ACKNOWLEDGMENT: Thanks to Noro Ramahatafandry for providing much of the data for this paper. 
1. For discussion of the rules for deriving the surface forms from their underlying representations, see Keenan \& Polinsky (1998), Erwin (1996), Paul $(1996 \mathrm{a} / \mathrm{b})$. Here I will mention only that when a consonant occurs at the end of a phonological word, an epenthetic $a$ is inserted after it, in accordance with a surface ban on closed syllables (e.g., velar-an > velarana).

2. Note that the TT suffixes -in and -an are generally treated not as separate voice markers, but as lexically-conditioned allomorphs of a single marker. However, Rahajarizafy (1960) notes that there are a handful of verbs which can take either suffix, with a concomitant difference in argument structure (see Pearson 2001 for discussion). Ileana Paul (p.c.) informs me that her consultants reject Rahajarizafy's examples, allowing a stem to take -in or -an in the TT form, but not both. Since my speaker accepts Rahajarizafy's examples, I will treat -in and -an as separate morphemes-while acknowledging that the distinction between them may be disappearing for some speakers.

3. Notice that in the case of (35), the Asp head is null, rather than surfacing as $-i n$, as expected. To capture this, I posit a morphological filter which blocks -in from attaching to a verb stem which already carries a suffix.

4. Because a clause can contain multiple PPs, I must assume that the higher applicative projection can be recursive. (It is possible that the lower applicative projection is recursive as well, although no more than projection will contain a DP specifier interpreted as an affected object.)

\section{References}

Carnie, Andrew, Heidi Harley, and Elizabeth Pyatt. 2000. VSO ordering as raising out of IP? Some evidence from Old Irish. The syntax of verb initial languages, ed. by Andrew Carnie and Eithne Guilfoyle, 39-59. Oxford: Oxford University Press.

Chomsky, Noam. 1999. Derivation by phase. MIT Occasional Papers in Linguistics 18. Cambridge, MA: MITWPL.

Chomsky, Noam. 2000. Minimalist inquiries: the framework. Step by step: Essays in minimalist syntax in honor of Howard Lasnik, ed. by Roger Martin, David Michaels, and Juan Uriagereka, 89-155. Cambridge, MA: MIT Press.

Chung, Sandra. 1998. The design of agreement: evidence from Chamorro. Chicago: University of Chicago Press.

Chung, Sandra. and James McCloskey. 1987. Government, barriers and smallclauses in Modern Irish. Linguistic Inquiry 18.173-237.

Dryer, Matthew. 1986. Primary objects, secondary objects, and antidative. Language 62.808-845.

Erwin, Sean. 1996. Quantity and moras: an amicable separation. The structure of Malagasy, vol. I, ed. by Matthew Pearson and Ileana Paul, 2-30. Los Angeles: UCLA Department of Linguistics.

Guilfoyle, Eithne. 2000. Tense and N-features in Irish. The syntax of verb initial languages, ed. by Andrew Carnie and Eithne Guilfoyle, 61-73. Oxford: Oxford University Press. 
Guilfoyle, Eithne, Henriette Hung, and Lisa Travis. 1992. Spec of IP and Spec of VP: two subjects in Austronesian languages. Natural Language and Linguistic Theory 10.375-414.

Keenan, Edward. 1995. Predicate-argument structure in Malagasy. Grammatical relations: Theoretical approaches to empirical questions, ed. by Clifford Burgess, Katarzyna Dziwirek, and Donna Gerdts, 171-216. Stanford: CSLI.

Keenan, Edward, and Maria Polinsky. 1998. Malagasy morphology. Handbook of morphology, ed. by Andrew Spencer and Arnold Zwicky, 563-623. Oxford: Oxford University Press.

Larson, Richard. 1988. On the double object construction. Linguistic Inquiry 19.335-392.

Marantz, Alec. 1993. Implications of asymmetries in double object constructions. Theoretical Aspects of Bantu Grammar, ed. by Sam Mchombo, 113-150. Stanford: CSLI.

Paul, Ileana. 1996a. The active marker and nasals in Malagasy. The Structure of Malagasy, vol. I, ed. by Matthew Pearson and Ileana Paul, 49-57. Los Angeles: UCLA Department of Linguistics.

Paul, Ileana. 1996b. The Malagasy genitive. The Structure of Malagasy, vol. I, ed. by Matthew Pearson and Ileana Paul, 76-91. Los Angeles: UCLA Department of Linguistics.

Paul, Ileana. 1999. Malagasy clause structure. Doctoral dissertation, McGill University.

Pearson, Matt. 2001. The clause structure of Malagasy: A minimalist approach. Doctoral dissertation, UCLA.

Pearson, Matt. to appear. The Malagasy subject/topic as an A'-element. Natural Language and Linguistic Theory.

Rahajarizafy, Antoine. 1960. Essai de grammaire malgache. Antanimena Tananarive: Imprimerie Catholique.

Sportiche, Dominique. 1992. Clitics, voice, and spec-head licensing. GLOW Newsletter 28.46-47.

Travis, Lisa. 1991. Derived objects, inner aspect, and the structure of VP. Paper presented at NELS 22.

Travis, Lisa. 1994. Event phrase and a theory of functional categories. Proceedings of the 1994 Annual Conference of the Canadian Linguistics Association, ed. by Päivi Koskinen, 559-569. Toronto: University of Toronto.

Matt Pearson

Linguistics Program, Reed College

3203 SE Woodstock Blvd

Portland, OR 97202

USA

matthew.pearson@reed.edu 